\title{
Video Article \\ Vagus Nerve Stimulation As an Adjunctive Neurostimulation Tool in Treatment-resistant Depression
}

\author{
Sebastian Moeller ${ }^{1,2}$, Caroline Lücke ${ }^{2}$, Christian Heinen ${ }^{3}$, Bettina H. Bewernick ${ }^{2,4}$, Merve Aydin ${ }^{2}$, Alexandra P. Lam² ${ }^{2}$, Teja W. Grömer ${ }^{5}$, \\ Alexandra Philipsen², Helge H.O. Müller ${ }^{2}$ \\ ${ }^{1}$ Universitätsklinik für Psychiatrie und Psychotherapie, Carl von Ossietzky Universität Oldenburg \\ ${ }^{2}$ Universitätsklinikum Bonn AöR, Klinik und Poliklinik für Psychiatrie \\ ${ }^{3}$ Universitätsklinik für Neurochirurgie am Evangelischen Krankenhaus Oldenburg, Carl von Ossietzky Universität Oldenburg \\ ${ }^{4}$ Klinik für Neurodegenerative Erkrankungen und Gerontopsychiatrie, Abteilung für medizinische Psychologie, Klinik für Psychiatrie und Psychotherapie, \\ Universitätsklinikum Bonn \\ ${ }^{5}$ Psychiatrische und Psychotherapeutische Klinik, Universitätsklinikum Erlangen
}

Correspondence to: Sebastian Moeller at sebastian.moeller@uni-oldenburg.de

URL: https://www.jove.com/video/58264

DOI: doi:10.3791/58264

Keywords: Medicine, Issue 143, Vagus nerve stimulation (VNS), therapy-resistant depression, major depression, neurostimulation, practical clinical management, tuning parameters, vagus nerve stimulator

Date Published: 1/7/2019

Citation: Moeller, S., Lücke, C., Heinen, C., Bewernick, B.H., Aydin, M., Lam, A.P., Grömer, T.W., Philipsen, A., Müller, H.H. Vagus Nerve Stimulation As an Adjunctive Neurostimulation Tool in Treatment-resistant Depression. J. Vis. Exp. (143), e58264, doi:10.3791/58264 (2019).

\section{Abstract}

Vagus nerve stimulation (VNS) is an approved neurostimulation therapy. The purpose of the method is to treat patients with therapy-resistant depression (TRD). VNS exhibits antidepressive and stabilizing effects. This method is particularly useful as a long-term treatment, in which up to two-thirds of patients respond. The vagus nerve stimulator is positioned on the left vagus nerve during a surgical procedure and is activated telemetrically by a wand connected to a handheld computerized device. The treating physician can perform various adjustments of the vagus nerve stimulator during in-office visits (e.g., by modifying stimulation intensity or stimulation frequency) to achieve maximum therapeutic effects with low side effects. Set-up of the device usually takes several months. Typical side effects include wound infection, temporary salivation, coughing, paralysis of the vocal cords, bradycardia, or even asystole. The patient can stop the VNS by placing a magnet over the generator. The current protocol describes delivery of the specific stimulation tool and methods for adjusting the tuning parameters to achieve the best remission rates in patients with TRD.

\section{Video Link}

The video component of this article can be found at https://www.jove.com/video/58264/

\section{Introduction}

Major depressive disease (MDD) is a frequently recurring or chronic psychiatric disease with high prevalence $e^{1,2}$ and is characterized by alterations in affect and mood. Of utmost importance is the association of MDD with high rates of suicidality and mortality ${ }^{3,4,5,6}$. Pharmacological treatment strategies include monoamine neurotransmitter reuptake inhibition [e.g., treatment with selective serotonin reuptake inhibitors (SSRIs) and serotonin-norepinephrine reuptake inhibitors (SNRIs)], lithium augmentation, or atypical antipsychotic augmentation strategies ${ }^{7,8}$. In addition to pharmacological treatment, psychotherapeutic approaches and supplemental noninvasive strategies are used to treat $\mathrm{MDD}^{8,9,10} \mathrm{Alternative}$ strategies such as light-based therapy, exercise, acupuncture, and yoga have also been successfully used in treatment of MDD ${ }^{8,9,11,12}$; although, these are not approved methods. However, up to $30-50 \%$ of all patients with MDD do not achieve remission with currently available treatment options $^{13}$. This subtype of MDD is called therapy-resistant depression (TRD) ${ }^{14,15,16}$. TRD is defined as the failure of two successive trials of antidepressant treatment administered at an appropriate dose and for a sufficient duration ${ }^{17,18,19}$.

The high rate of TRD poses a challenge in the overall management of patients with MDD ${ }^{13}$; thus, innovative add-on therapies for TRD are urgently needed. Neurostimulation tools such as electroconvulsive therapy (ECT), transcranial direct current stimulation (tDCS), repetitive transcranial magnetic stimulation (rTMS), deep brain stimulation (DBS), magnetic seizure therapy (MST), cranial electrotherapy (CES), and vagus nerve stimulation (VNS) are clinically available and promising tools in the psychiatric armamentarium for patients with TRD ${ }^{20}$. VNS is particularly useful in TRD and exhibits antidepressant and mood stabilizing effects. During long-term treatment ( $>12$ months), up to two-thirds of patients have responded favorably ${ }^{21,22,23}$. The American Psychiatric Association recommends the use of VNS as a treatment option after four treatment failures ${ }^{22}$.

In this study, we outline a protocol for clinicians, describing how to use and set up the VNS instrument for the continuous treatment of TRD. Additional notes on hardware components are included to help readers choose the product that best suits a patient's needs. 


\section{Protocol}

The following protocol provides a step-by-step manual for using VNS in the continuous treatment of TRD. The protocol is mainly based on the experiences and recommendations of the authors from different psychiatric centers in Germany. The protocol follows the guidelines of our institution's human research ethics committee.

\section{Pre-operative Examination}

NOTE: Before performing pre-operative steps, patients must be evaluated for solid compliance and willingness to attend regular (and likely irregular) control and tuning visitations after the implantation. In particular, aspects of compliance after implantation require careful consideration.

1. Contraindications

1. Before implantation, exclude contraindications for VNS therapy such as acute suicidality, substance addiction, and documented and difficult-to-treat non-compliance or non-adherence during previous psychiatric and/or somatic treatments. NOTE: Bipolar disorders and psychotic depressive symptoms are not contraindications for VNS therapy.

\section{Neurosurgical examination}

1. After positive psychiatric indication and evaluation, refer the probands to surgical evaluation to determine contraindications from a surgical perspective, then evaluate the eligibility for general anesthesia.

\section{Surgical Procedure}

1. Implantation

1. Administer general endo-tracheal anesthesia to the patient using standard techniques. Use propofol (4-12 mg/kg/h iv) for general anesthesia. Add an analgesic drug (e.g., remifentanyl at $1 \mu \mathrm{g} / \mathrm{kg} / \mathrm{min}$ iv) and a muscle relaxant (e.g., rocuronium at $0.6 \mathrm{mg} / \mathrm{kg}$ iv).

2. Position the patient supine on the operating table. Turn the head slightly to the right. Prepare the left side of the neck for supraclavicular lead implantation and an infraclavicular or axillar approach for the generator.

1. Use a specific agent for skin preparation to avoid surgical side infection. Scrub the skin with an aqueous-based iodophor such as povidone-iodine (PVP-I).

2. Perform an axillary incision by following a skin line. After hemostasis, bluntly dissect the subcutaneous fat to free the pectoralis major fascia and form a space for the generator.

3. Make an oblique skin incision of $4 \mathrm{~cm}$ approximately halfway between the mastoid and clavicle, with the medial border of the sternocleidomastoid muscle at the center. Then, divide the platysma muscle and dissect the cervical fascia.

4. Palpate the carotid artery to safely identify the correct neuro-vascular compartment. After opening the deep layer of the cervical fascia, carefully retract the carotid artery and internal jugular vein. The vagus nerve can be found between and below the vessels.

NOTE: At this point, we usually continue the procedure with operating microscopes.

5. Dissect the vagus nerve longitudinally for approximately $4-5 \mathrm{~cm}$, carefully sparing all branches and avoiding damage to the vasa nervorum.

1. Bring the lead in place from infra- to supra-clavicular using the tunneller device, enabling implantation.

6. Carefully wrap the inferior anchor tether of the VNS lead around the caudal aspect of the vagus nerve, then fix both positive and negative contact electrodes at the nerve. Insert the connector pin into the generator infraclavicularly.

7. After testing (see below), fix the lead with the tie-downs, facilitating a "spare loop". This allows the patient to move the head without tethering the probe. Suture the generator to the pectoralis major muscle fascia.

NOTE: It is important to place the electrodes inferior to the cardiac branches of the vagus nerve to avoid cardiac side effects.

2. Intraoperative lead testing

NOTE: It is necessary to first perform an intraoperative (lead) test, which should exclude the most common and serious side effects. Most notably, asystole can occur when the VNS is activated intraoperatively.

1. In situ testing

1. Connect the programming wand of the VNS while maintaining sterility intraoperatively in situ. Perform lead testing with a minimum of 10 seconds of stimulation of $0.5-1.0 \mathrm{~mA}$ and $25 \mathrm{~Hz}$. If no serious cardiac side effects occur, proceed to impedance testing.

3. Intraoperative impedance testing interpretation

1. Check the impedance during the in situ testing of the device. Check repeatedly.

NOTE: High impedances $(>1,700-2,000 \Omega)$ indicate poor contact between the helical electrodes and the nerve. During this phase, the anesthesiologist should be prepared for the occurrence of bradycardia or even asystole. Impedance is tested for a maximum of five seconds with an output current of $2.00-3.00 \mathrm{~mA}$ and should be under $100 \Omega$.

4. Fixing the electrodes after implantation

1. Use a tunneller to position the stimulator lead by passing it from the subcutaneous thoracic pocket into the cervical incision.

2. Tunnel the wire, and finally, secure the loops around the nerve to reduce the probability of dislodging the electrode.

\section{Finishing the operative procedures}


1. Create a strain relief bend to provide slack during movements of the neck.

2. Secure the electrode by tacking nonabsorbable sutures to silicone head holders at the deep cervical fascia and near the sternocleidomastoid muscle.

3. Fix the battery to the lead electrode and anchor it with nonabsorbable sutures in the fascia. Close the incisions by standard techniques.

\section{Post-surgical Treatment and Dosing}

1. Discharge

1. Discharge the patient one day after surgery, following an X-ray to ensure regular positioning of the device.

2. VNS dosing

1. Commence regular VNS dosing 2 weeks after implantation using the recommended stimulation settings $(1.5-3.0 \mathrm{~mA} ; 500 \mu \mathrm{s}$ pulse width; $20-30 \mathrm{~Hz} ; 30 \mathrm{~s}$ on, 5 min off), which must be gradually reached with $0.25-0.5 \mathrm{~mA}$ increases per week.

2. Start dosing (recommended) at $0.25 \mathrm{~mA}$ intervals as needed, and increase up to $2.00-3.00 \mathrm{~mA}$. Most patients (>90\%) will require dosing no higher than $2.00 \mathrm{~mA}$.

3. Dosing in cases of persisting depressive symptoms

1. Set the on/off time to $30 \mathrm{~s}$ on $/ 5 \mathrm{~min}$ off.

2. End dosing when a response to VNS is achieved, after a maximum of 9-12 months.

4. Dosing alternatives in cases of non-response or partial response

1. In cases of non-response or partial response, as an alternative to increasing the output current (mA), change the signal frequency (Hz) tuning (typically by decreasing from 30 to $20 \mathrm{~Hz}$ ) or reduce the off time to $3 \mathrm{~min}$.

5. Dosing in cases of side effects

1. If side effects occur (mainly laryngopharyngeal dysfunction such as hoarseness, dyspnea, and coughing), which tend to be transitory and directly associated with stimulation of the inferior (recurrent) laryngeal nerve, reduce the maximum mA tuning (typically no lower than $0.75 \mathrm{~mA}$ ) and/or change the on/off timing.

NOTE: An additional overview of the protocol is provided in Table 2.

\section{Representative Results}

The outcome measurements of VNS effectiveness usually include a decline in depressive symptoms, measured by observer-rated scales and/or self-rated assessment instruments [e.g., the Hamilton Depression Rating Scale, the Montgomery-Åsberg Depression Rating Scale, or the Beck Depression Inventory $(\mathrm{BDI})]^{6}$.

The first trial that systematically examined VNS in patients with major depressive episodes was conducted by Rush et al. ${ }^{24}$, who used VNS as an augmentation strategy with a 10 week combination VNS treatment alongside patients' normal medication. The average baseline 28 item Hamilton Depression Rating Scale score was 38.0. Forty percent of patients responded favorably, while $17 \%$ showed remission ${ }^{24}$. Sackheim et al. ${ }^{25}$ combined the initial study cohort with an additional 30 patients who were monitored for twelve weeks. The authors observed a response rate of $30 \%$ and a remission rate of $15 \%$. Schlaepfer and colleagues examined the antidepressive effects of VNS in an open, uncontrolled European multicenter study. Response and remission rates were $37 \%$ and $17 \%$, respectively ${ }^{26}$. Notably, in long-term follow-ups, an increase in both response and remission rates could be observed (Figure 1) ${ }^{23,27,28}$. In a sham-controlled, multicenter study, no significant difference between active and sham groups was observed ${ }^{29}$. However, the authors only performed ten weeks of active or sham VNS. Again, follow-up observations with higher remission and response rates suggest that VNS treatment has a beneficial effect over time ${ }^{30,31}$. The TRD registry followed the clinical course and outcome for TRD patients treated with and without adjunctive VNS ${ }^{22}$. In the observational study of unipolar or bipolar depression, the outcomes of 500 patients with VNS and 300 with treatment as usual (TAU) patients were compared. The subjects were permitted to choose between VNS and TAU at the time of screening. Registry results indicated that the adjunctive VNS group had better clinical outcomes than the TAU group. The 5 year cumulative response rate $(67.6 \%$ compared to $40.9 \%)$ and remission rate $(43.3 \%$ compared to $25.7 \%)$ were significantly higher in the VNS + TAU group than in the TAU-only group (Figure 2 and Figure 3) ${ }^{22}$. Moreover, the findings in the literature suggest decreased suicide attempts, reduced levels of suicidal ideation, and fewer hospitalizations due to depression in patients with VNS than in those with the same severity of disease but who are taking medications ${ }^{22,32}$.

In the following, we present three clinical cases to highlight the potential benefits as well as possible difficulties of providing VNS therapy to patients with TRD. 
A 53 year-old woman with a 20 year history of TRD was referred to our office. Her treatment included ineffective trials of tricyclic antidepressants, serotonin reuptake inhibitors, serotonin-norepinephrine reuptake inhibitors, and adjunctive treatments with antipsychotic agents and anxiolytics. She had also received a series of ECT. Although suicidality initially remitted, she remained depressed and required further ECT. ECT was continued, but her improved mood was unstable and did not result in a meaningful decrease in antidepressant medications. Her psychopathological characteristics included feelings of worthlessness, low self-esteem, and a daily lack of energy and motivation. The patient's long course of treatment-resistant major depressive episodes made her a candidate for VNS therapy. The patient underwent VNS therapy device implantation by a neurosurgeon during a $1.5 \mathrm{~h}$ procedure. The VNS was checked for functionality upon insertion. Stimulation was reactivated during an office visit within several weeks. At that time, the patient additionally received sertraline (200 mg/day). During the first stimulation visit, the vagus nerve stimulator was adjusted to $1.00 \mathrm{~mA}, 25 \mathrm{~Hz}$, and $30 \mathrm{~s}$ on/5 min off. During the second office visit, the dosing was increased to $1.50 \mathrm{~mA}$, and multiple $0.25 \mathrm{~mA}$ increases were performed. The patient did not report any side effects. Within three months, the current had been titrated up to an output of $1.5 \mathrm{~mA}$, with a standard signal frequency of $30 \mathrm{~Hz}$, pulse width of $500 \mu \mathrm{s}$, and signal time of 30 $\mathrm{s}$ on/5 min off. However, at that time, the patient experienced only minor improvements in mood and quality of life. The BDI showed a slight reduction of depression. The current output was set at $2.25 \mathrm{~mA}$, and during the following two weeks, an improvement was observed. We then added lithium at a dosage of $900 \mathrm{mg} /$ day to her medications and decreased the VNS off time to 2 min. During the next three months, the patient described an improvement of her mood and energy levels. Her BDI sum score showed a remission (i.e., the patient no longer met the criteria for major depression). Fortunately, she did not experience adverse events or side effects. Her medication was reduced to sertraline (100 mg/day). Perhaps the most significant improvement since the device adjustment was the reduction in medication use. The patient is now maintained on antidepressant therapy with sertraline and lithium. The device settings have not been changed again. After successful adjustment of the vagus nerve stimulator, the patient now participates every six months for control visits.

A 58-year-old chronically depressed patient was referred to our office. She had experienced ongoing depressive episodes since the age of 15 , requiring several courses of ECT. With each relapse of severe depression, she presented in a characteristic way, namely with periods of emotional non-response, unresponsiveness, an expressionless face, and staring eyes. When depressed, she would not care for herself, resulting in poor oral intake and rapid weight loss. She also socially disengaged from people and refused to take medication. She received several sessions of emergency ECT and showed a good response. During the last ECT session, the patient again showed marked improvement. Her BDI score was 46 before ECT and 18 afterwards. However, she refused to undergo maintenance ECT. She reported experiencing severe headache and memory loss after ECT. The patient's long and excellent response to ECT in terms of antidepressant effects made her a candidate for VNS therapy ${ }^{22}$. The patient underwent VNS therapy device implantation. The VNS was checked for functionality when it was inserted. Stimulation was reactivated during an office visit later that month. At the time of VNS initiation, the patient received citalopram (30 mg/day) and mirtazapine (60 mg/day). During this visit, the vagus nerve stimulator was adjusted to $1.00 \mathrm{~mA}$. Within five months, the current had been titrated up to an output of $2 \mathrm{~mA}$, standard signal frequency of $25 \mathrm{~Hz}$, pulse width of $250 \mu \mathrm{s}$, and signal time of $30 \mathrm{~s}$ on/5 min off. Six months after implantation of the VNS device, the patient was taking several antidepressants, including lithium and citalopram. The device was implanted 13 years ago. Since then, the patient's depressive symptoms have remained stable. She has reported subjective well-being and no side effects of VNS.

A 62-year-old man was referred to our office with a 40 year history of TRD. Due to the severity of the disease, he had been a patient in psychiatric hospitals and had made several severe suicide attempts. His depressive symptoms included melancholic mood, anhedonia, loss of appetite with consecutive weight loss, decreased energy, hopelessness, nihilistic delusion, and suicidal intentions. Somatic comorbidities consisted of controlled hypothyroidism and controlled epilepsy. The first seizure occurred after a closed head trauma when he was 49 years old. The patient had on average 3 seizures per year (tonic-clonic) between his 49th and 53th birthdays. Over the previous years, the patient had received treatments with different combinations of 2-5 antidepressants (e.g., citalopram, paroxetine, fluvoxamine, imipramine, amitriptyline, and venlafaxine extended release) without complete remittance. Remittance also failed with antidepressant augmentation trials with lithium, aripiprazole, and olanzapine. He also had 19 courses of ECT (bilateral and unilateral) during the period from 1980 to 2005 . The ECT effect was mostly short-lasting, as no sustained or long-term benefit from ECT was observed. He then underwent implantation with a VNS device for TRD in 2010. Previously, his prescribed medications (i.e., duloxetine, quetiapine, lithium, and topiramate) were unchanged. The vagus nerve stimulator was checked for functionality at the time of insertion. Stimulation was reactivated during an office visit later that month. During that visit, the vagus nerve stimulator was adjusted to $1.75 \mathrm{~mA}$. At the second in-office visit, dosing was increased to $2.00 \mathrm{~mA}$. The patient reported no side effects. Within 14 weeks, the current had been titrated up to $2.5 \mathrm{~mA}$ with a standard signal frequency of $30 \mathrm{~Hz}$, pulse width of $500 \mu \mathrm{s}$, and signal time of $30 \mathrm{~s}$ on $/ 5 \mathrm{~min}$ off. However, during an in-office visit, the patient reported an unusual globus sensation and hoarseness. Depressive symptoms markedly improved. The BDI score before implantation was 46 , but only 15 at 14 weeks post-implantation. Because of the remarkable antidepressant effect of VNS in this patient, we only made minor changes to the VNS setup. Moreover, the patient did not have any seizures after implantation of the VNS device. During the following three visits, which occurred over 2 weeks, we adapted the output to $2 \mathrm{~mA}$ and signal frequency to $40 \mathrm{~Hz}$ and adopted an extraordinary off time of $8 \mathrm{~min}$. With this setup, mild side effects occurred, but the antidepressant effect persisted. In accordance with the patient's wishes, we did not change the device settings.

These three patients, as well as our other patients receiving VNS, were prepared as required so that individual needs were addressed within the first six months after device implantation. After the first 6 months, follow-up appointments should be scheduled after 9,12 , and 24 months, or when an adjustment of dosing or device maintenance is necessary. The setup must be adapted to the patient's psychopathology, compatibility, psychological examination, somatic status (including history of side effects), laboratory, ECG, and (if necessary) imaging results. 


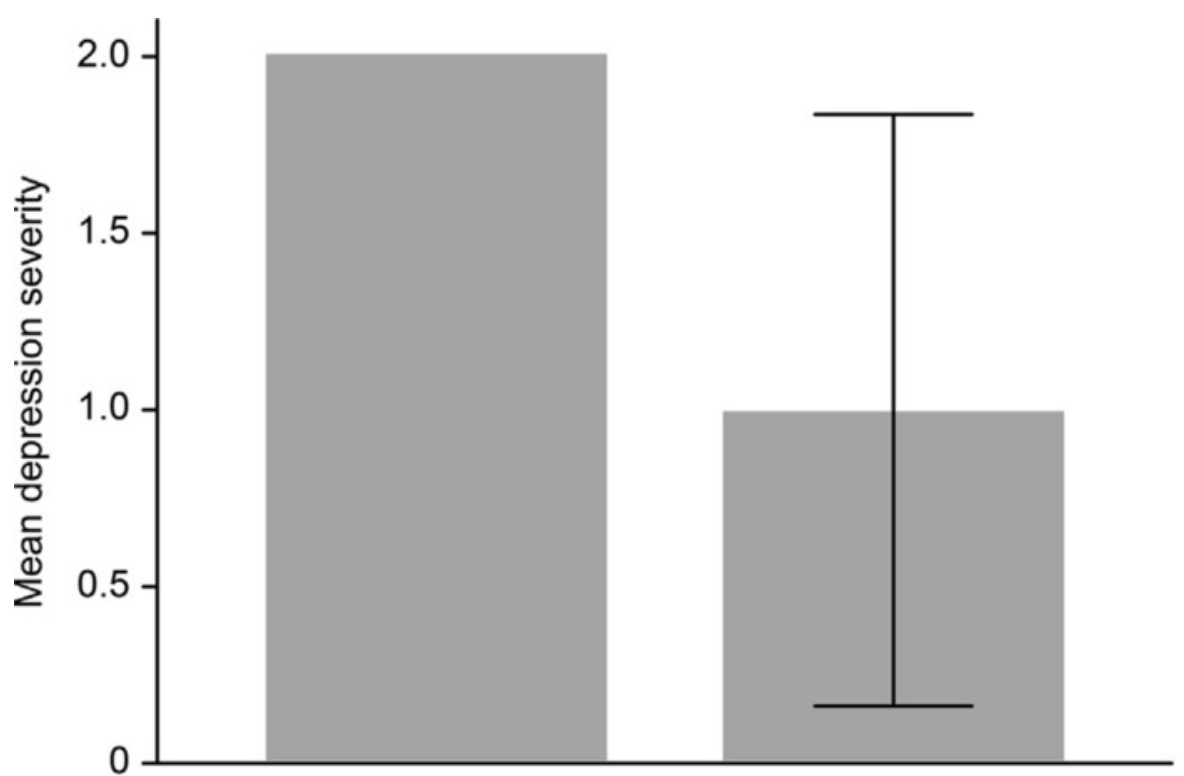

\section{Before VNS stimulation Under VNS stimulation}

Figure 1: Development of depression severity $(p<0.001)$ under VNS treatment compared to baseline (before VNS stimulation). The depression severity is coded as: $0=$ no depression, $1=$ mild depression, $2=$ moderate depression, and $3=$ severe depression. Treatment varied between 3 and 200 months (mean 104.9 months). This figure has been modified from Muller et al. (2017) ${ }^{23}$ with permission. The sample size was 18 (6 female, 12 male; mean age 54). Hamilton Depression Rating Scale raw scores were translated into an ordinal classification of depression severity. Differences in depression severity (pre- and post-treatment) were compared using a paired sample $t$-test. Pearson's productmoment correlation coefficient was computed to assess the relationship between VNS stimulation parameters and depression severity. Please click here to view a larger version of this figure.

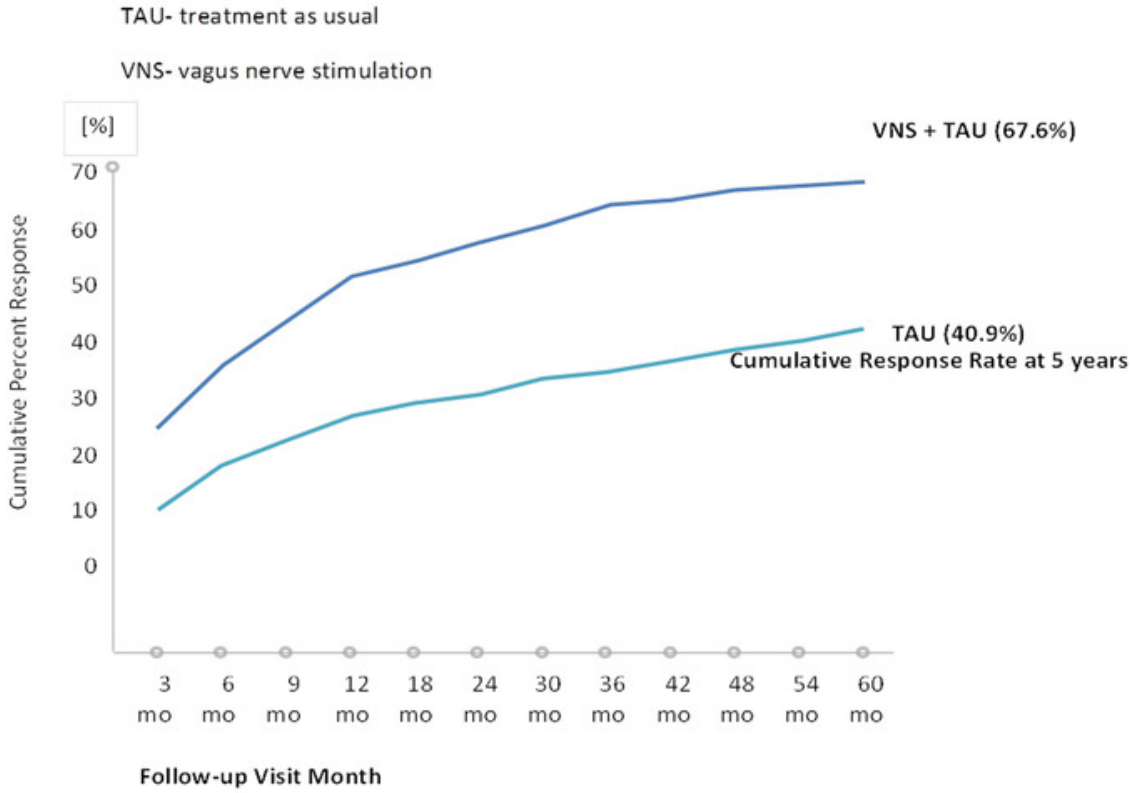

Figure 2: First-time response rate after 5 years based on the Montgomery-Åsberg Depression Rating Scale (MADRS). This figure has been modified with permission from Aaronson et al. ${ }^{22}$. A total of 765 patients (489 in the VNS group and 276 in the TAU group) was included in the efficacy analyses. Please click here to view a larger version of this figure. 


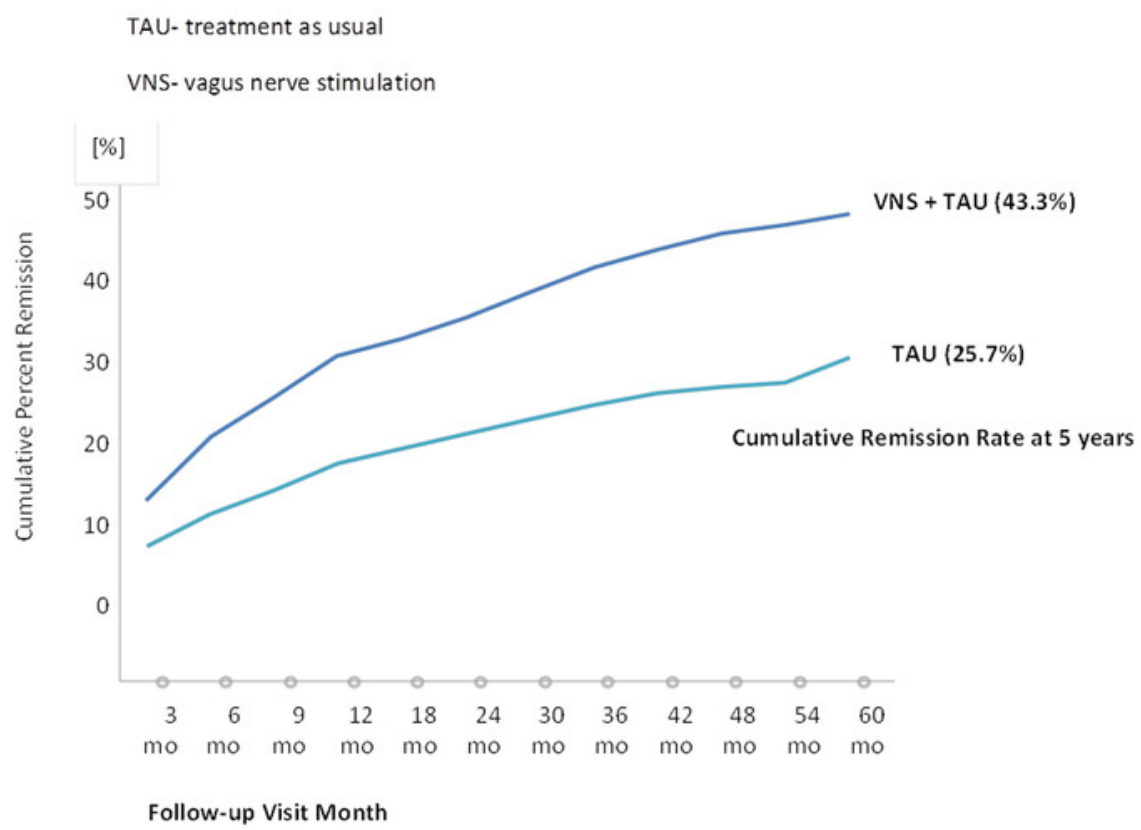

Figure 3: First-time remission rate based on the MADRS ( $\leq 9)$. This figure has been modified with permission ${ }^{22}$. A total of 765 patients $(489$ in the VNS group and 276 in the TAU group) was included in the efficacy analyses. Please click here to view a larger version of this figure.

\begin{tabular}{|c|c|c|}
\hline Adverse event, $\mathbf{n}(\%)$ & Year $1(\mathrm{~N}=700)$ & Year $2(\mathrm{~N}=344)$ \\
\hline Voice alteration & 485 (69.29) & $179(52.03)$ \\
\hline Dyspnea & $211(30.14)$ & $71(20.64)$ \\
\hline Pain & $199(28.43)$ & $41(11.92)$ \\
\hline Increased cough & $185(26.43)$ & $47(13.66)$ \\
\hline Incision pain & $181(25.86)$ & $15(4.36)$ \\
\hline Paresthesia & $159(22.71)$ & $39(11.34)$ \\
\hline Headache & $153(21.86)$ & $29(8.43)$ \\
\hline Neck pain & $139(19.86)$ & 55 (15.99) \\
\hline Pharyngitis & $122(17.43)$ & $25(7.27)$ \\
\hline Depression & $121(17.29)$ & $46(13.37)$ \\
\hline Dysphagia & $115(16.43)$ & $32(9.30)$ \\
\hline Incision-site reaction & $113(16.14)$ & $15(4.36)$ \\
\hline Nausea & 107 (15.29) & $12(3.49)$ \\
\hline Device-site pain & $98(14.00)$ & $11(3.20)$ \\
\hline Hypertonia & $92(13.14)$ & $31(9.01)$ \\
\hline Device-site reaction & $82(11.71)$ & $27(7.85)$ \\
\hline Insomnia & $75(10.71)$ & $22(6.40)$ \\
\hline
\end{tabular}

Table 1: Adverse events $\left[n_{33}(\%)\right]$ reported during the first and second years on VNS treatment. This table has been modified with permission from Berry et al. ${ }^{33}$. 


\begin{tabular}{|l|l|l|l|l|l|}
\hline Parameter & Units & Range & Target Range & $\begin{array}{l}\text { Maximum } \\
\text { Therapeutic Dosing }\end{array}$ \\
\hline Output Current & Milliampere $(\mathrm{mA})$ & $0-3.50$ & $1.0-2.0$ & 2.5 & $\begin{array}{l}\text { Stepwise increase } \\
\text { after first dosing } \\
\text { test (lead-test), } 0.25 \\
\text { every two weeks (if } \\
\text { tolerated) }\end{array}$ \\
\hline Signal Frequency & Hertz $(\mathrm{Hz})$ & $1-30$ & & 20 & $\begin{array}{l}\text { Modulation range } \\
\text { 20-30 Hz (for } \\
\text { antidepressive mode } \\
\text { of action) }\end{array}$ \\
\hline Pulse Width & & & & $20-30$ & \\
\hline Signal On-Time & Meconds $(\mathrm{s})$ & $7-60$ & 30 & 500 & $\begin{array}{l}\text { Decrease when side- } \\
\text { effects occur }\end{array}$ \\
\hline Signal Off-Time & Minutes (min) & $0.2-180$ & & $\begin{array}{l}\text { Increase when side- } \\
\text { effects occur }\end{array}$ \\
\hline
\end{tabular}

Table 2: Dosing recommendation for VNS. Normal-mode stimulation: stimulation $24 \mathrm{~h} /$ day and 7 days/week. Dosing is optimized for therapeutic effect and/or tolerability.

\section{Discussion}

VNS is a neurostimulation tool for chronic depression and TRD in patients aged 18 years or older who do not respond to other antidepressant treatments. VNS has been approved for use in the European Union and the United States ${ }^{22}$. VNS has been shown to be effective as an adjunctive treatment in TRD ${ }^{22}$; moreover, it has antisuicidal effects and improves quality of life ${ }^{22}$.

In this article, we present a protocol and additional information to help clinicians properly implement VNS in patients with TRD. The treating psychiatrist is responsible for the optimal dosing of the vagus nerve stimulator and for considering all aspects of the therapy, including the safety, tolerability, and efficacy of VNS in patients with TRD.

VNS requires an implantable pulse generator, which is surgically inserted under the skin of the chest, as described in the protocol. The VNS therapy pulse generator for depression treatment is placed subcutaneously in the left chest wall. Standard wound care is recommended during the first week after the procedure ${ }^{34,35}$. The repeated stimulation of the vagus nerve sends impulses from the peripheral nervous system, where the electrode is placed to the brain. The negative electrode generates action potentials that travel afferently via sensory fibers, while efferently traveling action potentials are mostly blocked by the positive electrode. Unblocked action potentials can cause side effects. A distance of approximately eight millimeters between the negative and positive electrodes is recommended ${ }^{36}$.

It is suggested not to use the right vagus nerve because of the risk of severe bradycardia and arrhythmias. In contrast, no such side effects have been reported when the left vagus nerve was used; however, several patients have had right-sided implants with good results. Surgical implantation is performed by means of minor surgery (mainly neurosurgical) ${ }^{37,38}$. The most frequent acute complications of vagus nerve stimulator implantation include temporary salivation, coughing, paralysis of the vocal cords, and lower facial weakness. Bradycardia occurs rarely, and asystole occurs very rarely. The risk for infection at the VNS site can be approximated to be between 1.1 and $3.9 \%{ }^{36}$.

Regarding psychiatric adverse effects, the rate of stimulation-induced switch to mania or hypomania in the VNS trials was low (i.e., $<0.01 \%$ at one year), and those symptoms diminished after modifying the stimulation parameters ${ }^{29}$. Side effects are generally fully reversible ${ }^{37,39}$. Adverse events reported during the first and second years on VNS therapy are summarized in Table $1^{33}$.

During surgery, the vagus nerve stimulator is regularly switched on to $0.5 \mathrm{~mA}$ to check whether the device is working properly and to avoid side effects such as asystole. After functionality has been verified, the device is switched off again. At least two weeks (depending on the potential side effects of tuning the VNS) after the device has been implanted, the vagus nerve stimulator is switched on again. Normal-mode stimulation is applied $24 \mathrm{~h}$ per day.

The stimulation amplitude is optimized with respect to the therapeutic effect and/or tolerability. The device is activated telemetrically by a wand connected to a handheld computerized device during psychiatric in-office visits. In general, the dose (i.e., the amplitude) is set to a level that the patient can tolerate (see Table 2). Adjustment of the treatment parameters, including output current, signal frequency, pulse width, signal on time, and signal off time, is non-invasively performed using the external interface. Importantly, the patient can stop the VNS stimulation by placing a magnet over the generator. After the magnet is removed, the generator restarts in normal-mode stimulation. The normal-mode output current can be increased to a therapeutic range as quickly as tolerable. More frequent visits (approximately 1 to 2 visits per week) at the beginning of the stimulation are recommended. Usually, stimulation is increased by $0.25 \mathrm{~mA}$ per visit. However, multiple $0.25 \mathrm{~mA}$ increases may be made at a single visit to reach the therapeutic range more quickly. Nevertheless, it is essential to ensure patient tolerance before making additional adjustments. The therapeutic range is usually between 1.0 and $2.0 \mathrm{~mA}$. In some patients, it is necessary to use higher output currents to receive additional efficacy. The effect of VNS is based on distinct mechanisms and is related to the anatomy of the vagus nerve.

The vagus nerve has anatomical connections, including those to the nucleus tractus solitaries, locus coeruleus, raphe nuclei, amygdala, hypothalamus, and orbitofrontal cortex ${ }^{40}$. VNS can increase the metabolic activity in the main serotonin- and noradrenalin-producing 
nuclei, resulting in augmented neurotransmitter concentrations in the $\mathrm{CSF}^{41,42}$. Thus, the mode of action of VNS works analogously to most antidepressants.

Neuroimaging studies have shown that activity in the thalamus and cortex of depressed patients is changed by VNS therapy. Changed activity in the orbital and ventromedial prefrontal cortices has also been recorded ${ }^{43,44}$. Several imaging studies have suggested that VNS therapy is associated with decreased metabolic activity in the right hemisphere and increased metabolic activity in the left hemisphere, thus attenuating the hemispheric imbalance in depressive patients ${ }^{40,45,46,47}$. Vagal afferents express IL-1 $\beta$ receptors that convey inflammatory signals to the hypothalamus, leading to a release of hypophysis adreno-corticotrophin hormones. This pathway also stimulates glucocorticoid release by the adrenal glands via a decrease in peripheral inflammation processes. In the vaso-vagal-inflammatory reflex loop, vagal afferents activate vagal efferents, which release acetylcholine $(\mathrm{ACh})$ at the distal end of the vagus nerve, inhibiting the release of pro-inflammatory cytokines such as $\mathrm{TNFa}^{48}$. In summary, the short- and medium-term antidepressant effects of VNS may be related to the increased availability of serotonin and noradrenalin, similar to the mechanism of antidepressive medication ${ }^{49}$. Long-term VNS has different effects than short-term VNS. The longterm antidepressant effects of VNS may be related to mitigation of the interhemispheric imbalance associated with depression (i.e., right-sided inhibition and left-sided activation $)^{45}$. Moreover, VNS may reduce inflammation that contributes to depression ${ }^{50}$, though not all the mechanisms of action of VNS are completely understood yet. The effects are thought to be gradual and acting with latency; consequently, VNS is usually not indicated for the relief of acute depressive symptoms ${ }^{32}$.

Up to $60 \%$ of patients with TRD show reduction in their depressive symptoms ${ }^{22,23,49,51,52}$. Long-term follow-ups also indicate fewer suicide attempts and lower levels of suicidal ideations and thoughts. Also documented are fewer hospitalizations in patients treated with VNS in addition to pharmacotherapy compared to patients with the same level of depression symptom severity who were only taking medications ${ }^{22,29,53}$.

Antidepressant and relapse prevention effects can even be observed after a long-term follow-up of 5 years ${ }^{22,23}$. Recently, in a large sample of patients with TRD, Aaronson and colleagues have shown significantly higher 5 year cumulative response rates and significantly higher remission rates in patients treated with VNS compared to patients who were only TAU ${ }^{22}$. The best-responding group consisted of patients with a history of good responses to ECT before receiving VNS ${ }^{22}$. The number needed to treat (NNT) for VNS ranges from 4 to 10. Given the high level of treatment resistance in this population of patients, the NNT remains clinically significant ${ }^{32}$.

Therefore, the long-term results of VNS are distinctly promising, suggesting that VNS might be particularly useful for patients with chronic depression for whom treatment resistance is a challenge ${ }^{20}$. Thus, after approximately 3 to 6 months of VNS therapy, treatment results can be expected.

The common definition of TRD is treatment failure of at least two antidepressants ${ }^{17,18,19}$. However, most patients with TRD who are treated with VNS have undergone more than two treatment trials of antidepressant medication before device implantation ${ }^{22}$. For example, in the longitudinal study by Aaranson et al. $(2017)^{22}$, patients who were treated with VNS failed an average of 8 treatments. The findings suggest that VNS might also be particularly useful in much heavier states of TRD. Antidepressive VNS therapy is mostly based on modifications to the output current and signal frequency. Changing the settings of on/off time parameters usually leads to a reduction in side effects.

Similar to TRD, VNS has been evaluated for its possible use for other psychiatric diseases, such as bipolar major depressive disorder, Alzheimer's disease, schizophrenia, obsessive compulsive disorder, panic disorder, post-traumatic stress disorder, treatment-resistant rapid-cycling bipolar disorder, fibromyalgia, and Prader-Willi syndrome ${ }^{54}$. The efficacy of VNS for affective disorders has been clearly demonstrated $^{20,22,23,54}$; however, for other psychiatric conditions, there have not yet been reports of either no effect or only preliminary data on efficacy ${ }^{54}$. Thus, no final conclusion about the efficacy of VNS in these psychiatric indications can be made currently. However, there may be potential for future therapeutic approaches ${ }^{54}$. Moreover, VNS is not only in the psychiatric armamentarium, as it is a common treatment method for treatment-refractory epilepsy ${ }^{5}$. In epilepsy patients, the stimulation parameters of the vagus nerve stimulator are similar to the settings in patients with TRD.

Initially, mood improvement in epilepsy patients with VNS led to the conclusion that VNS might also be useful in affective disorders ${ }^{32}$. VNS has also been assessed for the treatment of tinnitus ${ }^{56}$, inflammatory bowel diseases ${ }^{57}$, heart failure ${ }^{58}$, rheumatoid arthritis ${ }^{59}$, and many other conditions. Although preliminary data are promising, future evaluations are necessary for understanding the possible mechanisms and therapeutic potential in various clinical conditions and diseases.

Nevertheless, approximately one-third of patients with TRD do not adequately respond to VNS ${ }^{23}$. Moreover, the abovementioned side effects of VNS can limit its therapeutic action ${ }^{32}$. However, in cases of insufficient response or intolerable side effects, clinicians can modify the setup of the vagus nerve stimulator. Fine-tuning of the vagus nerve stimulator offers various possibilities for generating desirable responses to different medical conditions. Future perspectives to improve VNS efficacy and reduce side effects, such as including increasing the battery life, shortening the response time, transcutaneous stimulation, and identification of prognostic factors for valuable responses, should be further investigated.

\section{Disclosures}

Sebastian Moeller received a research grant from LivaNova, Inc. Christian Heinen received a speaker's honorarium from LivaNova, Inc. Bettina $\mathrm{H}$. Bewernick received a speaker's honorarium from LivaNova, Inc. and was member of an adivsory board of LivaNova, Inc. Merve Aydin, Alexandra Philomena Lam, Teja W. Grömer, Caroline Lücke, and Alexandra Philipsen have no competing interests. Helge H.O. Müller served as an advisory board member of LivaNova, Inc. and received speaker's compensation from LivaNova, Inc.

\section{Acknowledgements}

This study was financially supported by LivaNova, Inc. 


\section{References}

1. Beaucage, C., Cardinal, L., Kavanagh, M., Aube, D. Major depression in primary care and clinical impacts of treatment strategies: a literature review. Sante Mentale au Quebec. 34 (1), 77-100 (2009).

2. Maske, U. E., et al. Current major depressive syndrome measured with the Patient Health Questionnaire-9 (PHQ-9) and the Composite International Diagnostic Interview (CIDI): results from a cross-sectional population-based study of adults in Germany. BMC Psychiatry. 15 (77), (2015).

3. Tesio, V., et al. Screening of depression in cardiology: a study on 617 cardiovascular patients. International Journal of Cardiology. 245, 49-51 (2017).

4. Slepecky, M., et al. Which psychological, psychophysiological, and anthropometric factors are connected with life events, depression, and quality of life in patients with cardiovascular disease. Neuropsychiatric Disease and Treatment. 13, 2093-2104 (2017).

5. Lasserre, A. M., et al. Prospective associations of depression subtypes with cardio-metabolic risk factors in the general population. Moecular Psychiatry. 22 (7), 1026-1034 (2017).

6. Vandeleur, C. L., et al. Prevalence and correlates of DSM-5 major depressive and related disorders in the community. Psychiatry Research. 250, (50-58) 1023. (2017).

7. Crismon, M. L., et al. The Texas Medication Algorithm Project: report of the Texas Consensus Conference Panel on Medication Treatment of Major Depressive Disorder. Journal of Clinical Psychiatry. 60 (3), 142-156 (1999).

8. Negt, P., et al. The treatment of chronic depression with cognitive behavioral analysis system of psychotherapy: a systematic review and meta-analysis of randomized-controlled clinical trials. Brain and Behavior. 6 (8), e00486 (2016).

9. Harter, M., et al. [Psychotherapy of depressive disorders: Evidence in chronic depression and comorbidities]. Nervenarzt. 89 (3), 252-262 (2018).

10. Thase, M. E., et al. Cognitive therapy versus medication in augmentation and switch strategies as second-step treatments: a STAR D report. American Journal of Psychiatry. 164 (5), 739-752 (2007).

11. Smith, C. A., Armour, M., Lee, M. S., Wang, L. Q., Hay, P. J. Acupuncture for depression. The Cochrane Database of Systematic Reviews. 3, CD004046 (2018).

12. Zhao, X., Ma, J., Wu, S., Chi, I., Bai, Z. Light therapy for older patients with non-seasonal depression: A systematic review and meta-analysis Journal of Affective Disorders. 232, 291-299 (2018).

13. Schlaepfer, T. E., et al. The hidden third: improving outcome in treatment-resistant depression. Journal of Psychopharmacology. 26 (5), 587-602 (2012).

14. Rush, A. J., et al. Report by the ACNP Task Force on response and remission in major depressive disorder. Neuropsychopharmacology. 31 (9), 1841-1853 (2006).

15. Rush, A. J., et al. Acute and longer-term outcomes in depressed outpatients requiring one or several treatment steps: a STAR ${ }^{\star} D$ report. American Journal of Psychiatry. 163 (11), 1905-1917 (2006).

16. Mojtabai, R. Nonremission and time to remission among remitters in major depressive disorder: revisiting STAR ${ }^{*} \mathrm{D}$. Depression and Anxiety. (2017).

17. Bschor, T. Therapy-resistant depression. Expert Review of Neurotherapeutics. 10 (1), 77-86 (2010).

18. Holtzmann, J., et al. [How to define treatment-resistant depression?]. La Presse Médicale. 45 (3), (2016).

19. Wiles, N., et al. Clinical effectiveness and cost-effectiveness of cognitive behavioural therapy as an adjunct to pharmacotherapy for treatmentresistant depression in primary care: the CoBalT randomised controlled trial. Health Technology Assessment. 18 (31), 1-167, vii-viii (2014).

20. Muller, H. H. O., et al. Vagus Nerve Stimulation (VNS) and Other Augmentation Strategies for Therapy-Resistant Depression (TRD): Review of the Evidence and Clinical Advice for Use. Frontiers in Neuroscience. 12, 239 (2018).

21. Carreno, F. R., Frazer, A. Vagal Nerve Stimulation for Treatment-Resistant Depression. Neurotherapeutics. 14 (3), $716-727$ (2017).

22. Aaronson, S. T., et al. A 5-Year Observational Study of Patients With Treatment-Resistant Depression Treated With Vagus Nerve Stimulation or Treatment as Usual: Comparison of Response, Remission, and Suicidality. American Journal of Psychiatry. 174 (7), $640-648$ (2017).

23. Muller, H. H. O., Lucke, C., Moeller, S., Philipsen, A., Sperling, W. Efficacy and long-term tuning parameters of vagus nerve stimulation in long-term treated depressive patients. Journal of Clinical Neuroscience. 44, 340-341 (2017).

24. Rush, A. J., et al. Vagus nerve stimulation (VNS) for treatment-resistant depressions: a multicenter study. Biological Psychiatry. 47 (4), 276-286 (2000).

25. Sackeim, H. A. et al. Vagus nerve stimulation (VNS) for treatment-resistant depression: efficacy, side effects, and predictors of outcome. Neuropsychopharmacology. 25 (5), 713-728 (2001).

26. Schlaepfer, T. E., et al. Vagus nerve stimulation for depression: efficacy and safety in a European study. Psychological Medicine. 38 (5), 651-661 (2008).

27. Nahas, Z., et al. Two-year outcome of vagus nerve stimulation (VNS) for treatment of major depressive episodes. Journal of Clinical Psychiatry. 66 (9), 1097-1104 (2005).

28. Bajbouj, M., et al. Two-year outcome of vagus nerve stimulation in treatment-resistant depression. Journal of Clinical Psychopharmacol. 30 (3), 273-281 (2010).

29. Rush, A. J., et al. Vagus nerve stimulation for treatment-resistant depression: a randomized, controlled acute phase trial. Biological Psychiatry. 58 (5), 347-354 (2005).

30. Rush, A. J., et al. Effects of 12 months of vagus nerve stimulation in treatment-resistant depression: a naturalistic study. Biological Psychiatry. 58 (5), 355-363 (2005).

31. Nierenberg, A. A., Alpert, J. E., Gardner-Schuster, E. E., Seay, S., Mischoulon, D. Vagus nerve stimulation: 2-year outcomes for bipolar versus unipolar treatment-resistant depression. Biological Psychiatry. 64 (6), 455-460 (2008).

32. Cusin, C., Dougherty, D. D. Somatic therapies for treatment-resistant depression: ECT, TMS, VNS, DBS. Biology of Mood \& Anxiety Disorders. 2, 14 (2012).

33. Berry, S. M., et al. A patient-level meta-analysis of studies evaluating vagus nerve stimulation therapy for treatment-resistant depression. Medical Devices (Auckland, N.Z.). 6, 17-35 (2013). 
34. Giordano, F., Zicca, A., Barba, C., Guerrini, R., Genitori, L. Vagus nerve stimulation: Surgical technique of implantation and revision and related morbidity. Epilepsia. 58 (Suppl 1), 85-90 (2017).

35. Al Omari, A. I., et al. The vagal nerve stimulation outcome, and laryngeal effect: Otolaryngologists roles and perspective. American Journal of Otolaryngology. 38 (4), 408-413 (2017).

36. Morris, G. L. III, et al. Evidence-based guideline update: vagus nerve stimulation for the treatment of epilepsy: report of the Guideline Development Subcommittee of the American Academy of Neurology. Neurology. 81 (16), 1453-1459 (2013).

37. Elliott, R. E., et al. Efficacy of vagus nerve stimulation over time: review of 65 consecutive patients with treatment-resistant epilepsy treated with VNS > 10 years. Epilepsy \& Behavior. 20 (3), 478-483 (2011).

38. Ng, W. H., Donner, E., Go, C., Abou-Hamden, A., Rutka, J. T. Revision of vagal nerve stimulation (VNS) electrodes: review and report on use of ultra-sharp monopolar tip. Child's Nervous System. 26 (8), 1081-1084 (2010).

39. Schneider, U. C., Bohlmann, K., Vajkoczy, P., Straub, H. B. Implantation of a new Vagus Nerve Stimulation (VNS) Therapy(R) generator, AspireSR(R): considerations and recommendations during implantation and replacement surgery--comparison to a traditional system. Acta Neurochirurgica (Wien). 157 (4), 721-728 (2015).

40. Pardo, J. V., et al. Chronic vagus nerve stimulation for treatment-resistant depression decreases resting ventromedial prefrontal glucose metabolism. Neuroimage. 42 (2), 879-889 (2008).

41. Roosevelt, R. W., Smith, D. C., Clough, R. W., Jensen, R. A., Browning, R. A. Increased extracellular concentrations of norepinephrine in cortex and hippocampus following vagus nerve stimulation in the rat. Brain Resesarch. 1119 (1), 124-132 (2006).

42. Hassert, D. L., Miyashita, T., Williams, C. L. The effects of peripheral vagal nerve stimulation at a memory-modulating intensity on norepinephrine output in the basolateral amygdala. Behavioral Neuroscience. 118 (1), 79-88 (2004).

43. Muller, H. H., Reulbach, U., Maler, J. M., Kornhuber, J., Sperling, W. Facilitative effects of VNS on the motor threshold: implications for its antidepressive mode of action? Journal of Neural Transmission (Vienna). 120 (10), 1507-1510 (2013).

44. Chae, J. H., et al. A review of functional neuroimaging studies of vagus nerve stimulation (VNS). Journal of Psychiatric Research. 37 (6), 443-455 (2003).

45. Conway, C. R., et al. Association of cerebral metabolic activity changes with vagus nerve stimulation antidepressant response in treatmentresistant depression. Brain Stimulation. 6 (5), 788-797 (2013).

46. Kosel, M., Brockmann, H., Frick, C., Zobel, A., Schlaepfer, T. E. Chronic vagus nerve stimulation for treatment-resistant depression increases regional cerebral blood flow in the dorsolateral prefrontal cortex. Psychiatry Research. 191 (3), 153-159 (2011).

47. Nahas, Z., et al. Serial vagus nerve stimulation functional MRI in treatment-resistant depression. Neuropsychopharmacology. 32 (8), 1649-1660 (2007).

48. Bonaz, B., Sinniger, V., Pellissier, S. Anti-inflammatory properties of the vagus nerve: potential therapeutic implications of vagus nerve stimulation. Journal of Physiology. 594 (20), 5781-5790 (2016).

49. Furmaga, H., Shah, A., Frazer, A. Serotonergic and noradrenergic pathways are required for the anxiolytic-like and antidepressant-like behavioral effects of repeated vagal nerve stimulation in rats. Biological Psychiatry. 70 (10), 937-945 (2011).

50. Corcoran, C., Connor, T. J., O'Keane, V., Garland, M. R. The effects of vagus nerve stimulation on pro- and anti-inflammatory cytokines in humans: a preliminary report. Neuroimmunomodulation. 12 (5), 307-309 (2005).

51. George, M. S., et al. A one-year comparison of vagus nerve stimulation with treatment as usual for treatment-resistant depression. Biological Psychiatry. 58 (5), 364-373 (2005).

52. Shen, H., Fuchino, Y., Miyamoto, D., Nomura, H., Matsuki, N. Vagus nerve stimulation enhances perforant path-CA3 synaptic transmission via the activation of beta-adrenergic receptors and the locus coeruleus. International Journal of Neuropsychopharmacology. 15 (4), $523-530$ (2012).

53. Burke, M. J., Husain, M. M. Concomitant use of vagus nerve stimulation and electroconvulsive therapy for treatment-resistant depression. The Journal of ECT. 22 (3), 218-222 (2006).

54. Cimpianu, C. L., Strube, W., Falkai, P., Palm, U., Hasan, A. Vagus nerve stimulation in psychiatry: a systematic review of the available evidence. Journal of Neural Transmission (Vienna). 124 (1), 145-158 (2017).

55. Oliveira, T., Francisco, A. N., Demartini, Z. J., Stebel, S. L. The role of vagus nerve stimulation in refractory epilepsy. Arquivos de NeuroPsiquiatria. 75 (9), 657-666 (2017).

56. Peter, N., Kleinjung, T. Neuromodulation for tinnitus treatment: an overview of invasive and non-invasive techniques. The Journal of Zhejiang University Science B: Biomedicine \& Biotechnology. 12 (10), (2018).

57. Breit, S., Kupferberg, A., Rogler, G., Hasler, G. Vagus Nerve as Modulator of the Brain-Gut Axis in Psychiatric and Inflammatory Disorders Frontiers in Psychiatry. 9, 44 (2018).

58. Devgun, J., Jobanputra, Y. B., Arustamyan, M., Chait, R., Ghumman, W. Devices and interventions for the prevention of adverse outcomes of tachycardia on heart failure. Heart Failure Reviews. 12 (10), 018-9680 (2018).

59. Koopman, F. A., van Maanen, M. A., Vervoordeldonk, M. J., Tak, P. P. Balancing the autonomic nervous system to reduce inflammation in rheumatoid arthritis. Journal of Internal Medicine. 282 (1), 64-75 (2017). 